\title{
Imaging atom sites with near node
}

\section{photoelectron holography}

\author{
T. Greber, J. Wider, A. Verdini ${ }^{\dagger}, A$. Morgante ${ }^{\dagger}$, and J. Osterwalder
}

Physik Institut der Universität Zürich, Winterthurerstr. 190, CH-8057 Zürich, Switzerland

${ }^{\dagger}$ Laboratorio TASC, Istituto Nazionale per la Fisica della Materia, Basovizza SS14 Km 163.5, I-34012 Trieste, Italy

$R_{\mathrm{t}}^{\mathrm{e}}$ ecent photoelectron diffraction experiments at the synchrotron ELETTRA (Trieste) demonstrated a strong suppression of forward scattering in aluminium for $\mathrm{Al} 2 s$ photoelectrons $\left(E_{k i n} \approx 1 \mathrm{keV}\right)$ that are measured at an angle near the nodal plane of the outgoing $p$-wave [1]. This use of the selection rules for photoemission considerably improves the holographic reconstructions of real space from photoelectron diffraction data. These holographic reconstructions provide 3 dimensional images of atomic sites in unit cells with an extension of more than $10 \AA$.

Structure determination on the atomic level is of key importance for the understanding of physical and chemical processes. At surfaces low-energy electron diffraction (LEED) with kinetic energies between 20 and $500 \mathrm{eV}$ is the most successful technique for this purpose [2]. For real space structure determination LEED suffers, in spite of its obvious advantages, from three problems: It is not chemically sensitive, i.e. the individual atomic species cannot be identified directly, the electron scattering cross sections are large, and the phase information of the scattered wave is lost. In principle, photoelectron diffraction circumvents these problems. Core level photoemission is chemically sensitive, and in going to higher kinetic energies multiple scattering decreases roughly proportionally to the wavelength of the electrons. The phase problem, however, is common to all diffraction techniques, and most structure determination schemes are based on trial and error methods. If the phase of the scattered wave is known and the scattering can be treated in the kinematic approximation, i.e. without multiple scattering, the positions of the individual scatterers in real space may be recovered directly by Fourier transform.

Gabor's idea of holography is a way of recovering the phase information and gives, in principle, access to the full three dimensional structure [3]. Szöke extended holography to X-ray and electron diffraction experiments. His "inside source" concept (see Box 1) proposes that the X-rays or electrons which are created at atomic sites form a hologram in Gabor's sense [4]. After the emission process, the "coherent beam" splits into an unscattered reference wave $\Psi_{r}$ and a scattered object wave $\Psi_{0}$ that interfere in the detector. The measured intensity I(k) can thus be written as:

$$
\mathrm{I}(\mathbf{k})=\left|\Psi_{r}+\Psi_{o}\right|^{2}=\Psi_{r} \Psi_{r}^{*}+\Psi_{r} \Psi_{o}^{*}+\Psi_{o} \Psi_{r}^{*}+\Psi_{o} \Psi_{o}^{*},
$$

where $\mathbf{k}$ is the wave vector of the reference and the object waves. The intensities of a large set of $\mathbf{k}$ vectors constitute a hologram and permit the reconstruction of an image $U(r)$ in real space. If $r$ is known and the object self interference term o is small, $U(r)$ is determined by the Fourier transform of $\mathrm{I}(\mathbf{k})$, i.e. from the interference terms $\Psi_{r} \Psi_{o}^{*}+\Psi_{0} \Psi_{r}^{*}$. These interference terms contain an image and a twin image, and methods exist to distinguish the two [5].For three dimensional images, the k-space sample has to span three dimensions. This is achieved by scanning two emission angles and/or $|\mathbf{k}|$ (multiple energy sampling).
Inside source holography with X-rays [6] and its time reversed sister with $\gamma$-rays [7] have been demonstrated. These kinds of holography apply for heavy elements as scatterers and emitters and for a probed volume which is fairly large. Inside source holography with electrons is more sensitive, in particular to light elements, and applications with particular emitter geometries have been reported [8]. However, several problems are encountered: In the relevant electron kinetic energy range, the scattering cross section of electrons is of the order of $1 \AA^{2}$ and highly anisotropic. Therefore, multiple scattering is important and the scattering amplitudes and phase shifts are not isotropic. The dominant feature of this anisotropy is forward scattering, which is a consequence of the focusing by the attractive ion cores of an incoming electron wave along its $\mathbf{k}$-vector [9]. Forward scattering is a zero order diffraction feature that contains no holographic information, i.e. information on the path length differences

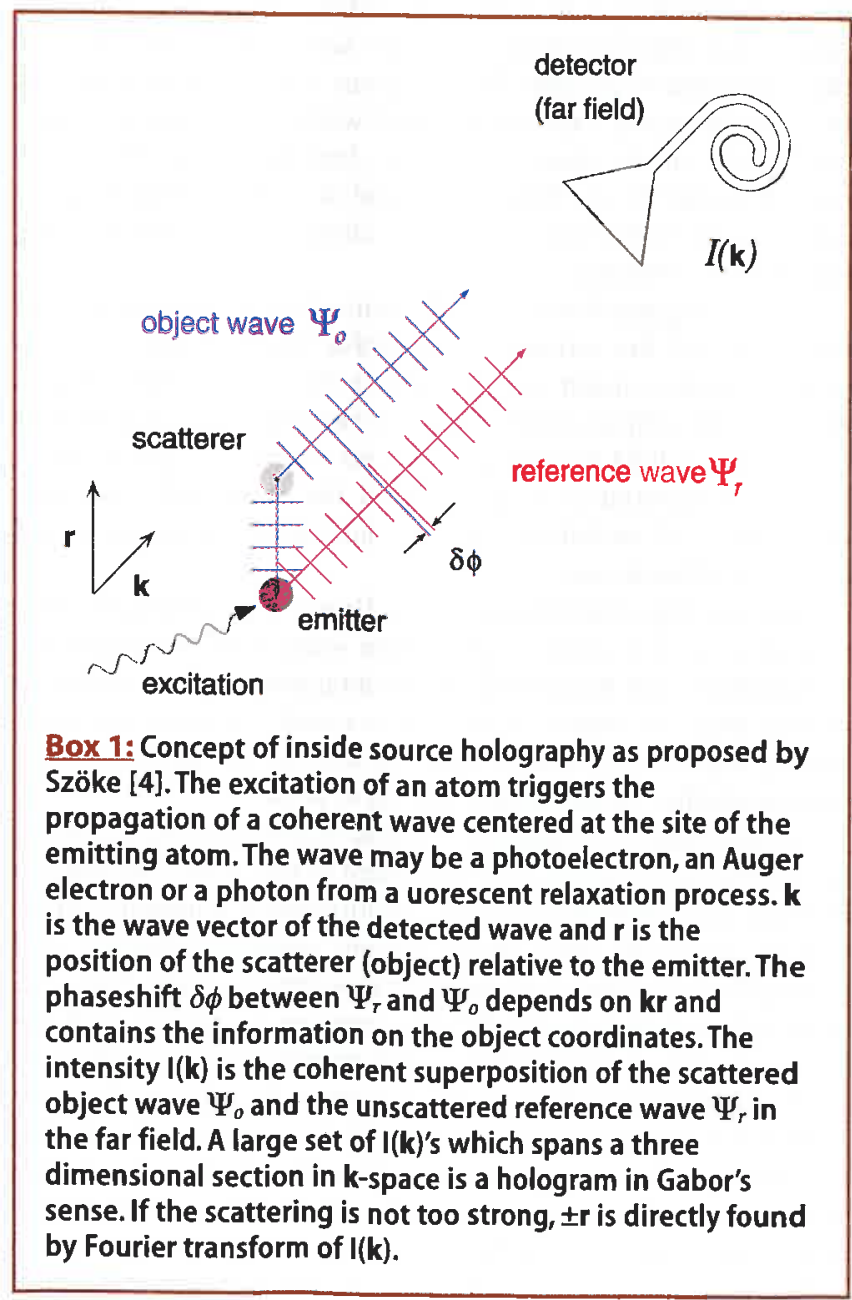


between the scattered and the unscattered waves. The application of Fourier transforms to recover the real space structure from photoelectron or Auger electron diffraction data is thus not straightforward.

At a first glance, forward scattering seems to be an intrinsic feature of photoelectron diffraction, at least if the emitter does not lie in the top layer. There is, however a twist around this problem if the anisotropic nature of the photoelectron source wave (see Box 2 ) is exploited [10]. In a single scattering picture, the photoelectron diffraction intensity $I(k)$ (Equation 1 ) may be written as:

$$
\mathrm{I}(\mathbf{k})=\left|\Psi_{\text {source }}(\boldsymbol{\vartheta} \equiv \mathbf{0}, \mathbf{R})+\sum_{i} \frac{f\left(\vartheta_{i}\right)}{\left|\mathbf{R}-\mathbf{r}_{i}\right|} \Psi_{\text {source }}\left(\vartheta_{i}, \mathbf{r}_{i}\right)\right|^{2},
$$

where the emitter (source) sits at the origin, $\mathbf{R}$ is the position of the detector (in the direction of $\mathbf{k}$ ), and $\mathbf{r}_{i}$ the location of the scatterer (object) i.f $\left(\vartheta_{i}\right)$ is the complex scattering amplitude, where $\vartheta_{i}$ is the angle between $r_{i}$ and $R . \Psi_{\text {soume }}(\vartheta \equiv 0, R)$ is the reference wave $\Psi_{r}$. It can be seen that the importance of forward scattering $\vartheta_{i}=0$ scales with the intensity of the reference wave. Therefore the Fraunhofer condition $\left|\Psi_{r}\right| \gg\left|\Psi_{o}\right|$ can generally not be optimized in electron holography since it would cause strong forward scattering. If the source wave is isotropic the relative weight of the forward scattering is constant. For anisotropic source waves, such as a $p$-wave that is created by the photo-excitation of an $s$-level, the relative importance of the reference wave and thus the weight of forward scattering can be tuned. If for example the electron emission direction lies on a node of the photoemission source wave, no forward scattering is expected. However, within the single scattering picture, such photoelectron diffraction patterns cannot be considered as holograms, since the reference wave is missing in this geometry. For $1 \mathrm{keV}$ electrons it was shown by scattering calculations that there is an optimum angle of about $10^{\circ}$ near the nodal plane of an outgoing $p$-wave, where the holographic reconstructions are best [10]. This angle is a compromise between the

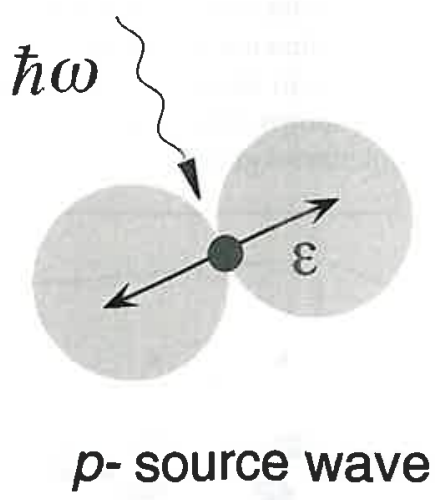

Box 2: Photoemission from a core level of an atom provides a highly coherent electron source wave that is localized on the emitting atom. The incoming photon breaks the spherical symmetry of the atom and creates an anisotropic electron source wave. The dipole selection rules dictate a change in angular momentum $h \Delta l$ between the initial state and the photoemission final state (source wave) of $\pm h$. For the excitation of a s-core level $(l=0)$ this results in the creation of a pure $p$-wave $(l=1)$ with a node. The emission from other levels than $s$-levels generally produces no nodes. The picture shows a $p$-wave as generated by light that is linearly polarized along the electric field vector $\varepsilon$. effect of the disturbing object self interference term $\Psi_{o} \Psi_{o}^{*}$ and that of the forward scattering. In Figure 1, the far node geometry is compared with the near node geometry. The Figure shows simulated photoelectron diffraction patterns and the corresponding holographic reconstructions for a single emitter and a single scatterer. In the near node geometry, the relative weight of the interference pattern is strongly enhanced compared to the forward scattering. The holographic reconstructions from the diffraction pattern in the far node geometry show "fingers" that correspond to the forward scattering cone in the photoelecton diffraction near field. On the other hand, the diffraction data in the near node geometry show "droplet"-features near the atomic site

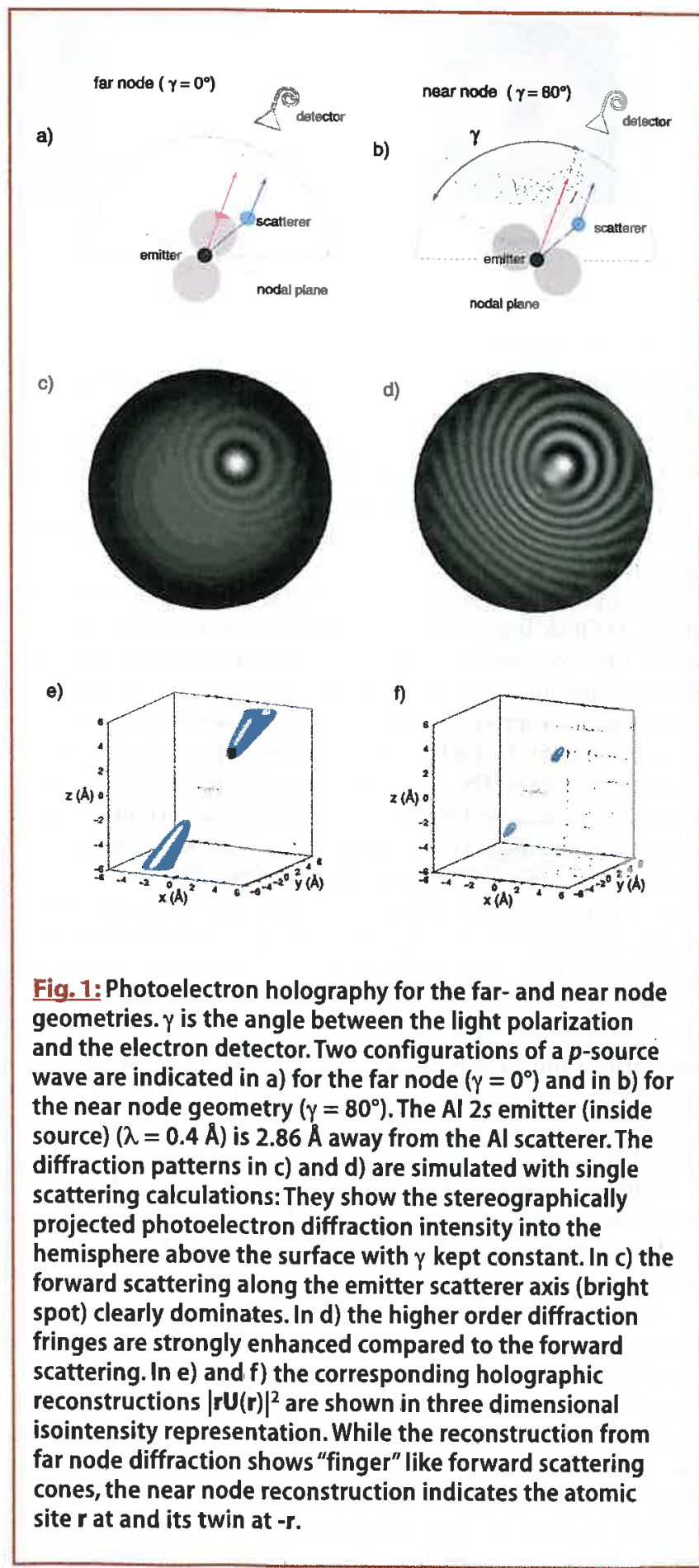



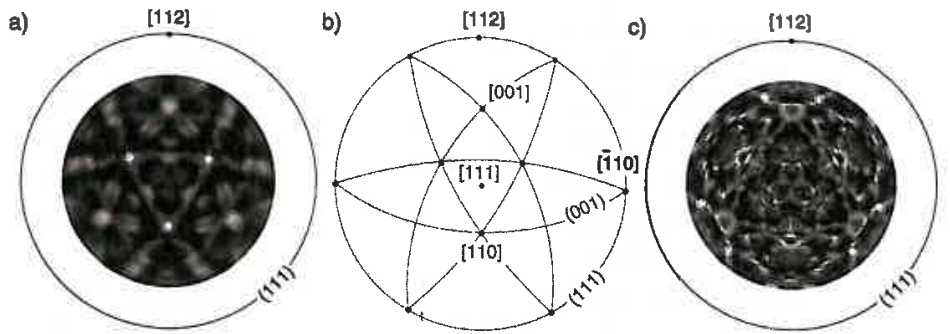

[1̄12]
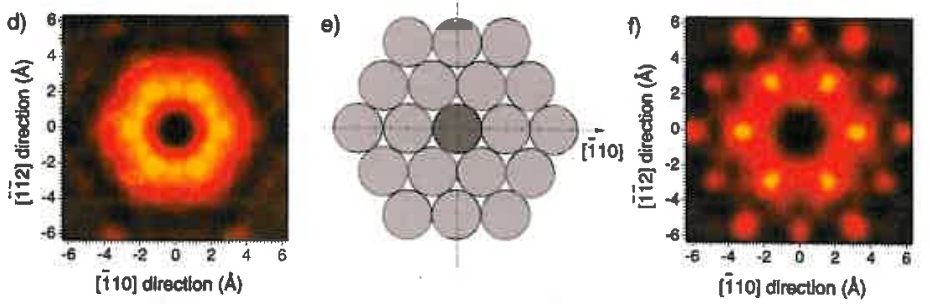

Fig. 2: Comparison of far node and near node photoelectron diffraction data and their holographic reconstructions. a) and c) Stereographically projected experimental AI $2 s$ $\left(E_{k i n}=942 \mathrm{eV}\right)$ photoelectron diffraction patterns from an Al (111) single crystal for the far node and the near node geometry. In the far node geometry (a) forward scattering dominates the pattern as can be seen from the stereographic projection of the high density crystal chains and planes in (b). In the near node diffraction pattern (hologram) no distinct forward scattering features are visible. d) and f) Corresponding holographic real space reconstructions of a plane parallel to the surface that contains the emitter (inside source) at $(0,0)$. They should show the expected image of an AI (111) plane (e). In the near node reconstruction ( $f$ ) nearest, next nearest and second next nearest neighbors are clearly resolved as local maxima while in the far node picture (d) no clear atom positions can be seen (from Ref. [1]). of the scatterer and its twin image. It has to be noted that near node photoelectron holography, requires a constant angle $\gamma$ between the detector and the light polarization. Otherwise the near node condition is not fulfilled for all emission directions. Therefore, in this kind of experiment, the sample has to be rotated with respect to the reference frame of the light source and the detector.

The experiments on the $\mathrm{Al}$ (111) surface have been performed at the ALOISA beamline at the synchrotron facility ELETTRA $[1,11]$. The face centered cubic $(f c c)$ structure of aluminium with a nearest neighbor distance of $2.86 \AA$, is well known. Furthermore, the inversion symmetry of the $f c c$ structure causes the twin image to coincide with the image. The experiments were performed at room temperature. The $\mathrm{Al} 2 s$ diffraction data sets contain about 1600 data points evenly spaced in $2 \pi / 3$ from normal emission down to a polar angle of $70^{\circ}$ and are recorded with a high angular resolution of $\Delta \Omega=1^{\circ}$. With the photon energy of $1070 \mathrm{eV}$ and the $118 \mathrm{eV}$ binding energy of the $\mathrm{Al} 2 s$ core level, the electron wavelength becomes $0.4 \AA$. Two orientations of the linear light polarization were used. In the near node geometry the angle $\gamma$ between the detector and the polarization was set to $80^{\circ}$ and in the far node geometry to $0^{\circ}$. The far node geometry data serve for comparison and an absolute determination of the crystal orientation. In order to remove the polar angle dependence of the instrumental response function, the azimuthal data sets (Fig. 2a and c) are normalized for every polar emission angle.

Figure 2 demonstrates near node photoelectron diffraction (and holography) with experimental data [1]. The angle-dependent $\mathrm{Al} 2 s$ photoemission intensities for the far node and the near node geometry are stereographically projected in Figures $2 \mathrm{a}$ ) and c). White corresponds to highest and black tolowest intensity. From comparison with Figure $2 b$ ) which shows the corresponding real space projection of an $f c c$ crystal onto the (111) plane, it is seen that the far node diffraction pattern can be regarded as a projection of nuclear charge along the nearest neighbour directions. The forward scattering focuses intensity along nearest neighbor directions and thus causes high intensity along low index atomic chains and planes [9]. This correspondence is not seen in the near node diffraction pattern (Figure $2 c$ ) and thus indicates suppres- sion of forward scattering. In order to get real space images of the emitter's environment, the diffraction patterns are holographically interpreted [12]. The image function $|\mathbf{r} U(\mathbf{r})|^{2}$ is shown after a convolution with a Gaussian with $1 \AA$ full width at half maximum, without any low intensity cut-off. In the near node geometry, the holographic reconstruction of real space around the emitter in the (111) plane clearly reveals the positions of the surrounding atoms. In Figure 2f) nearest, next nearest and even second next nearest neighbor positions are resolved as local maxima in the image. This is not the case for the far node geometry data, where instead of distinct atomic positions a "nearest neighbor belt" is found. As usual with inside source holography, the emitter sits at the origin of the image and is not reproduced since.

In Figure 3, a three dimensional image of the holographic reconstruction of the data in Figure $2 \mathrm{c}$ ) is displayed. The convoluted image function is shown within a sphere around the emitter with $8 \AA$ diameter. Intensities greater than $83 \%$ of the maximum

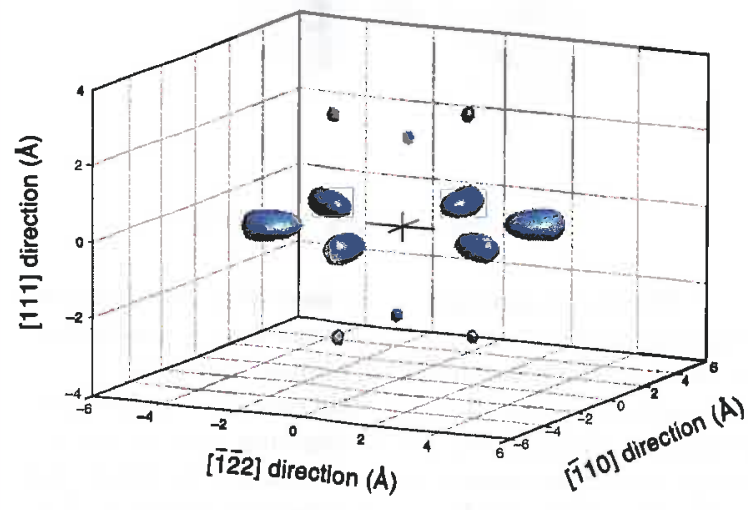

Fig. 3: Three dimensional isointensity representation of the holographic image as reconstructed from the photoelectron diffraction data in Figure $2 \mathrm{c}$ ). The atomic environment of an $\mathrm{Al} 2 \mathrm{~s}$ photoemitter at $(0,0,0)$ in $\mathrm{Al}(111)$ is shown inside a shell with $4 \AA$ radius (from Ref. [1]). 
intensity are displayed opaque. The image quality in the [111] direction, normal to the surface, is not as good as in the (111) plane. This can be partly understood by considering that the emitters in the top layers see truncated neighbor shells and that the thermal smearing is more pronounced normal to the surface. Furthermore, the $\mathbf{k}_{[111]}$ sampling is more limited in our experimental setup. However, from the data in Figure $2 c$ ) the three dimensional structure of the twelve nearest neighbors around the emitter is recovered. In Figure 3, the expected $\mathrm{ABC}$ stacking of subsequent (111) planes in the $f c c$ structure is correctly reproduced. The holographic image is, however, not very precise with respect to the absolute length. This is caused by the atomic scattering phase shifts that imply larger and anisotropic "effective" scattering paths. All nearest neighbor distances in Figure 3 are overestimated by about one wavelength, but in a fairly isotropic way. The twelve emitter-nearest neighbor distances scatter by $0.1 \AA$ around the mean value of $3.4 \AA$ (instead of the $2.86 \AA$ expected from the $f c c$ structure of aluminium).

These findings demonstrate that the atomic structure of molecular objects with a size of the order of $10 \AA$ can be explored with near node photoelectron holography. The chemical sensitvity of core level photoemission and the exploitation of the anisotropy of its source wave present an opportunity for structure determination in large unit cells with complicated molecular structures.

It is a pleasure to acknowledge the Instituto Nazionale per la Fisica della Materia (INFM) for beamtime and hospitality and the Swiss National Science Foundation for supporting this project.

\section{References}

[1] J. Wider, F. Baumberger, M. Sambi, R. Gotter, A. Verdini, E. Bruno, D. Cvetko, A. Morgante, T. Greber and J. Osterwalder, Phys. Rev. Lett. 86, 2337, (2001).

[2] http://electron.lbl.gov/ssd/

[3] D. Gabor, Nature, 161, 777, (1948).

[4] A. Szöke, in Short wavelength Coherent radiation: Generation and Applications (Eds. Attwood, D.T. and Boker, J.) 361-467 (AIP Conf. Proc. No.147, American Institute of Physics, New York, 1986).

[5] J.J. Barton, Phys. Rev. Lett., 67, 3106, (1991).

[6] M. Tegze, G. Faigel, S. Marchesini, M. Belakhovsky and A.I. Chumakov, Phys. Rev. Lett., 82, 4847, (1999).

[7] P. Korecki, J. Korecki, T. Ślezak, Phys. Rev. Lett. 79, 3518, (1997).

[8] H. Wu, G.J. Lapeyre, H. Huang and S.Y. Tong, Phys. Rev. Lett. 71, 251, (1993).

[9] C.S. Fadley, Surf. Sci. Rep., 19, 231, (1993).

[10] T. Greber and J. Osterwalder, Chem. Phys. Lett., 256, 653, (1996).

[11] http://tasc.area.trieste.it/tasc/lds/aloisa/aloisa.html

[12] A. Stuck, D. Naumović, H. A. Aebischer, T. Greber, J. Osterwalder and L. Schlapbach, Surf. Sci., 264, 380, (1992).

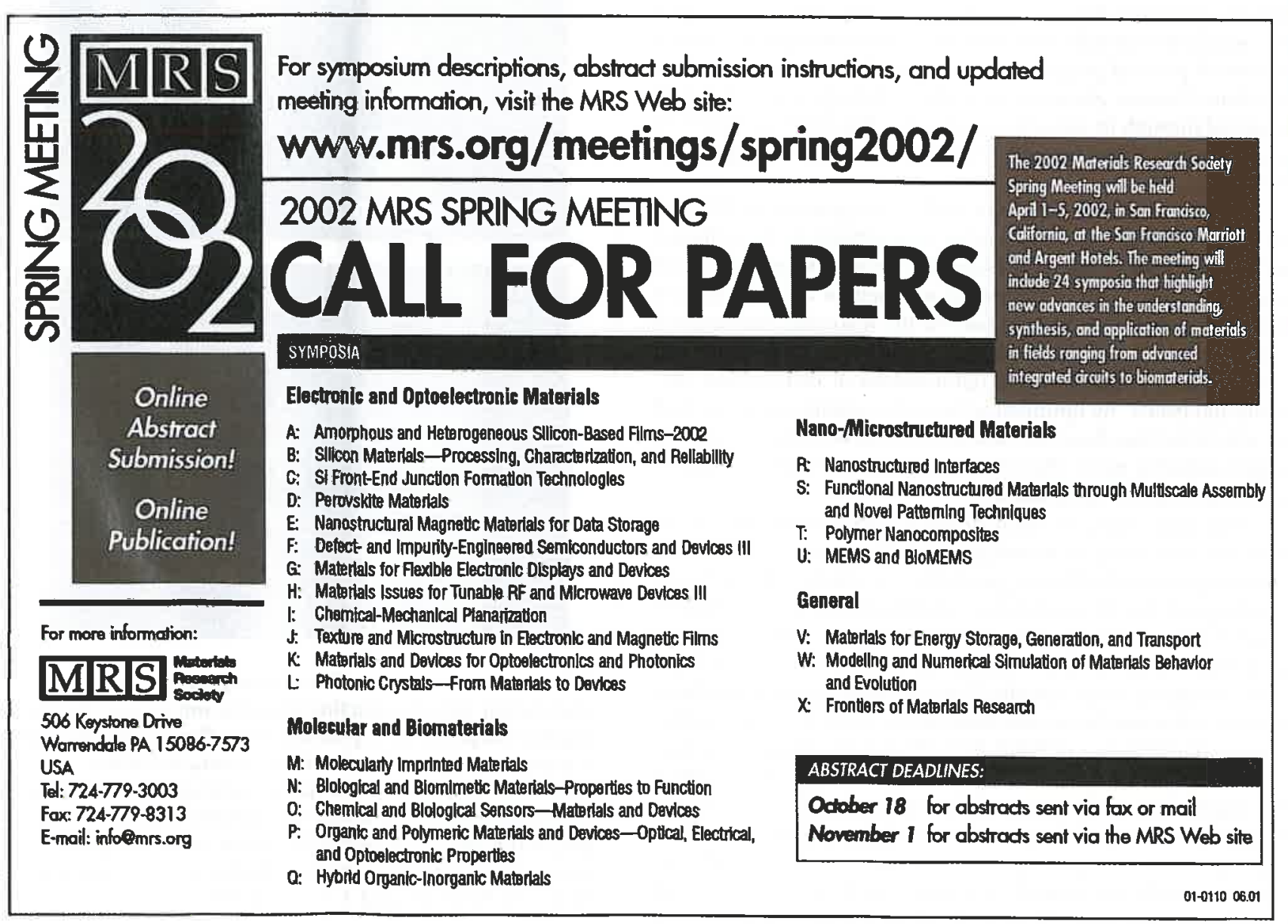

